TRANSACTIONS OF THE

AMERICAN MATHEMATICAL SOCIETY

Volume 360, Number 10, October 2008, Pages 5489-5507

S 0002-9947(08)04587-X

Article electronically published on May 28, 2008

\title{
CLASSIFICATION OF QUADRUPLE GALOIS CANONICAL COVERS I
}

\author{
FRANCISCO JAVIER GALLEGO AND BANGERE P. PURNAPRAJNA
}

Dedicated to Ignacio Sols

\begin{abstract}
In this article we classify quadruple Galois canonical covers of smooth surfaces of minimal degree. The classification shows that they are either non-simple cyclic covers or bi-double covers. If they are bi-double, then they are all fiber products of double covers. We construct examples to show that all the possibilities in the classification do exist. There are implications of this classification that include the existence of families with unbounded geometric genus, in sharp contrast with triple canonical covers, and families with unbounded irregularity, in sharp contrast with canonical covers of all other degrees. Together with the earlier known results on double and triple covers, a pattern emerges that motivates some general questions on the existence of higher degree canonical covers, some of which are answered in this article.
\end{abstract}

\section{INTRODUCTION}

Classification problems are of central importance in algebraic geometry. In the realm of algebraic surfaces, the geography of surfaces of general type, by far the largest class of surfaces, is much less charted and understood. An important subclass of surfaces of general type are those whose canonical map is a cover of a simpler surface, most notably, a surface of minimal degree. In the seventies and eighties Horikawa and Konno ( $[\mathrm{Ho},[\mathrm{KO})$ classified these covers when the degree of the cover is 2 and 3. In this article and in its sequel GP4 we classify surfaces of general type whose canonical map is a quadruple Galois cover of a surface of minimal degree.

Covers of varieties of minimal degree have a ubiquitous presence in various contexts. They appear in the classification of surfaces of general type $X$ with small $c_{1}^{2}$ and play an important role in mapping the geography of surfaces of general type. They are also the chief source in constructing new examples of surfaces of general type, as the work of various geometers illustrates. These covers occur as well in the study of linear series on important threefolds such as Calabi-Yau threefolds, as the work in BS, GP1 and DP shows. They also become relevant in the study of

Received by the editors November 15, 2006.

2000 Mathematics Subject Classification. Primary 14J10, 14J26, 14J29.

The first author was partially supported by MCT project number BFM2000-0621. He is grateful for the hospitality of the Department of Mathematics of the University of Kansas at Lawrence.

The second author is grateful to NSA and the GRF of the University of Kansas for supporting this research project. He is also grateful for the hospitality of the Departamento de Álgebra of the Universidad Complutense de Madrid.

(C)2008 American Mathematical Society 5489 Reverts to public domain 28 years from publication 
the canonical ring of a variety of general type, as can be seen in results from GP2] and $\mathrm{Gr}$.

Compared to the canonical morphism of a curve, the canonical morphism of a surface is much more subtle and allows a much wider range of possibilities due to the existence of higher degree covers. The degree of the canonical morphism of a curve is bounded by 2 . In contrast, results of Beauville show that the degree of the canonical morphism from a surface of general type $X$ onto a surface of minimal degree, or more generally, onto a surface with geometric genus $p_{g}=0$, is bounded by 9 if $\chi(X) \geq 31$ (see $\mathrm{Be}$ ).

Surfaces of minimal degree are classically known to be linear $\mathbf{P}^{2}$, the Veronese surface in $\mathbf{P}^{5}$ and rational normal scrolls, which can be smooth (these include the smooth quadric hypersurface in $\mathbf{P}^{3}$ ) or singular (these are cones over a rational normal curve). As pointed out before, the classification of the canonical covers of these surfaces is only complete when the degree of the cover is 2 and 3 . Horikawa also studied quadruple covers of linear $\mathbf{P}^{2}$. The next step in this classification is the study of quadruple covers of an arbitrary surface of minimal degree.

In this work we classify all quadruple Galois canonical covers of smooth surfaces of minimal degree $W$. In GP4 we classify quadruple Galois covers of $W$ when $W$ is singular. There are many interesting consequences of the classification done here and in GP4. Our classification yields, among other things, some striking contrasts with double and triple covers. Before we look at them we state the main result of this article:

Theorem 0.1. Let $X$ be a canonical surface and let $W$ be a smooth surface of minimal degree. If the canonical bundle of $X$ is base-point-free and $X \stackrel{\varphi}{\longrightarrow} W$ is a quadruple Galois canonical cover, then $W$ is either linear $\mathbf{P}^{2}$ or a smooth Hirzebruch surface $\mathbf{F}_{e}$, with $0 \leq e \leq 2$, embedded by $\left|C_{0}+m f\right|(m \geq e+1)$. Let $G$ be the Galois group of $\varphi$.

A) If $G=\mathbf{Z}_{4}$, then $\varphi$ is the composition of two double covers $X_{1} \stackrel{p_{1}}{\longrightarrow} W$ branched along a divisor $D_{2}$ and $X \stackrel{p_{2}}{\longrightarrow} X_{1}$, branched along the ramification of $p_{1}$ and $p_{1}^{*} D_{1}$, where $D_{1}$ is a divisor on $W$ and with trace zero module $p_{1}^{*} \mathcal{O}_{W}\left(-\frac{1}{2} D_{1}-\frac{1}{4} D_{2}\right)$.

B) If $G=\mathbf{Z}_{2}^{\oplus 2}$, then $X$ is the fiber product over $W$ of two double covers of $W$ branched along divisors $D_{1}$ and $D_{2}$, and $\varphi$ is the natural morphism from the fiber product to $W$.

More precisely, $\varphi$ has one of the sets of invariants shown in the following table. Conversely, if $X \stackrel{\varphi}{\longrightarrow} W$ is either

I) the composition of two double covers $X_{1} \stackrel{p_{1}}{\longrightarrow} W$, branched along a divisor $D_{2}$, and $X \stackrel{p_{2}}{\longrightarrow} X_{1}$, branched along the ramification of $p_{1}$ and $p_{1}^{*} D_{1}$, and with trace zero module $p_{1}^{*} \mathcal{O}_{W}\left(-\frac{1}{2} D_{1}-\frac{1}{4} D_{2}\right)$, with $D_{1}$ and $D_{2}$ as described in rows 1,3 and 5 of the table below; or

II) the fiber product over $W$ of two double covers $X_{1} \stackrel{p_{1}}{\longrightarrow} W$ and $X_{2} \stackrel{p_{2}}{\longrightarrow} W$, branched respectively along divisors $D_{2}$ and $D_{1}$, as described in rows 2, 4, 6,7 and 8 of the table below,

then $X \stackrel{\varphi}{\longrightarrow} W$ is a Galois canonical cover whose Galois group is $\mathbf{Z}_{4}$ in case $I$ and $\mathbf{Z}_{2}^{\oplus 2}$ in case II. 


\begin{tabular}{|c|c|c|c|c|c|c|}
\hline$W$ & $p_{g}(X)$ & $G$ & $D_{1} \sim$ & $D_{2} \sim$ & $q(X)$ & $c_{1}^{2} / c_{2}$ \\
\hline \hline $\mathbf{P}^{2}$ & 3 & $\mathbf{Z}_{4}$ & conic & quartic & 0 & $\frac{1}{11}$ \\
\hline $\mathbf{P}^{2}$ & 3 & $\mathbf{Z}_{2}^{\oplus 2}$ & quartic & quartic & 0 & $\frac{1}{11}$ \\
\hline $\mathbf{F}_{e}$ & $2 m-e+2$ & $\mathbf{Z}_{4}$ & $(2 m-e+1) f$ & $4 C_{0}+(2 e+2) f$ & 0 & $\frac{2 m-e}{4 m-2 e+9}$ \\
\hline $\mathbf{F}_{e}$ & $2 m-e+2$ & $\mathbf{Z}_{2}^{\oplus 2}$ & $2 C_{0}+(2 m+2) f$ & $4 C_{0}+(2 e+2) f$ & 0 & $\frac{2 m-e}{4 m-2 e+9}$ \\
\hline $\mathbf{F}_{0}$ & $2 m+2$ & $\mathbf{Z}_{4}$ & $(2 m+4) f$ & $4 C_{0}$ & 1 & $\frac{m}{2 m+3}$ \\
\hline $\mathbf{F}_{0}$ & $2 m+2$ & $\mathbf{Z}_{2}^{\oplus 2}$ & $2 C_{0}+(2 m+4) f$ & $4 C_{0}$ & 1 & $\frac{m}{2 m+3}$ \\
\hline $\mathbf{F}_{0}$ & $2 m+2$ & $\mathbf{Z}_{2}^{\oplus 2}$ & $(2 m+2) f$ & $6 C_{0}+2 f$ & $m$ & $\frac{2 m}{m+9}$ \\
\hline $\mathbf{F}_{0}$ & $2 m+2$ & $\mathbf{Z}_{2}^{\oplus 2}$ & $(2 m+4) f$ & $6 C_{0}$ & $m+3$ & 2 \\
\hline
\end{tabular}

Remark 0.2. Note that, whereas $W$ is always simply connected, the intermediate cover $X_{1}$ in A) and I) of Theorem 0.1 is sometimes simply connected and sometimes not. An example of the former is the case in row 1 of the above table, provided $D_{2}$ is smooth, and the case in row 3 , when $e=0,1$, provided again that $D_{2}$ is smooth (see [No]). An example of the latter is the case in row 5 ; in this case $\pi_{1}\left(X_{1}\right)=\mathbf{Z} \times \mathbf{Z}$, as $X_{1}$ is the product of a smooth elliptic curve and $\mathbf{P}^{1}$. That is why in order to determine $p_{2}$, in general, we need to specify not only the branch divisor but also the trace zero module of the cover.

Theorem 0.1 is split inside the paper into Corollary 2.5] and Theorems 4.1, 5.1, 5.2, 6.1 and 6.2. We also construct families of examples to show the existence of all the cases that appear in the classification. A notable fact that is not mentioned in the statement of the theorem is that there exist families of smooth surfaces of general type $X$ for every case described in rows $2,4,6,7$ and 8 of the above table (that is, all cases where $G=\mathbf{Z}_{2}^{\oplus 2}$ ). In comparison, we show that the quadruple cyclic canonical covers of smooth surfaces of minimal degree are always singular. We also show that quadruple cyclic canonical covers are non-simple cyclic.

One of the interesting implications of the main result of this article is the existence of families of quadruple canonical covers with unbounded geometric genus and the existence of families with unbounded irregularity. The unboundedness of the geometric genus is in sharp contrast with the situation of triple covers, and the unboundedness of the irregularity is in sharp contrast with the canonical covers of all other degrees. The geometric genus of canonical double covers is unbounded, but they are all regular, and even simply connected surfaces. The classification of triple covers by Konno shows that the geometric genus of canonical triple covers is bounded by 5 and that they are all regular. For quadruple Galois covers we show the existence of families of surfaces $X$ for each possible value of $p_{g}(X)$ (see rows 3 to 8 of the above table). We also show that there exist unbounded families of surfaces $X$ for each possible value of $q(X)$ (see rows 7 and 8 of the table).

The classification of quadruple covers provides other significant contrast with double and triple covers and clearly brings out the marked difference between even and odd degree covers. The only smooth targets of quadruple Galois canonical covers that occur are linear $\mathbf{P}^{2}$ and rational normal scrolls which correspond to only 
three Hirzebruch surfaces, namely $\mathbf{F}_{0}, \mathbf{F}_{1}$ and $\mathbf{F}_{2}$. In the case of canonical double covers, linear $\mathbf{P}^{2}$ and smooth rational scrolls corresponding to every Hirzebruch surface appear as an image of the canonical morphism. In the case of canonical triple covers, the list is reduced drastically and the only possible smooth target is linear $\mathbf{P}^{2}$.

The geography of Chern numbers of quadruple Galois canonical covers is markedly different from that of double and triple canonical covers. For double covers the ratio $c_{1}^{2} / c_{2}$ approaches $1 / 5$ as $p_{g}$ approaches $\infty, c_{1}^{2} / c_{2}$ always being less than $1 / 5$. In the case of triple covers, $c_{1}^{2} / c_{2}$ only takes three values, the largest of them being $1 / 7$. For both double and triple covers, $\left(c_{1}^{2}, c_{2}\right)$ lies well below the line $c_{1}^{2}=2 c_{2}$. By contrast, quadruple Galois covers have a much richer geography and traverse a larger region, as can be seen from the previous table. In particular, Chern pairs $\left(c_{1}^{2}, c_{2}\right)$ from different families approach two different lines, namely, $c_{1}^{2}=\frac{1}{2} c_{2}$ and $c_{1}^{2}=2 c_{2}$. The latter line is actually attained.

The classification of quadruple covers of singular targets in GP4 together with earlier results on double and triple covers exhibit a striking pattern. Indeed, we show that quadruple covers of singular targets form a bounded family with respect to both the geometric genus and irregularity as in the case of double and triples covers of singular targets. The results in this article and in GP4 predict a precise numerology, regarding $p_{g}$ and $q$, that might hold for higher degree covers. The following facts make it clear what we mean: there do not exist canonical covers of odd degree of smooth scrolls (see [GP2, Proposition 3.3]) and there do not exist Galois canonical covers $X \stackrel{\varphi}{\longrightarrow} W$ of prime degree $p>3$ of surfaces $W$ of minimal degree if $X$ is regular or if $W$ is smooth (see Theorem 7.2, Corollary 7.3 and GP2, Corollary 3.2]). This motivates us to pose a general question (see Question [7.4) on the non-existence of higher, prime degree Galois canonical covers of surfaces of minimal degree.

The classification obtained in this article and in GP4 has further applications. In a forthcoming paper we determine the ring generators of the quadruple covers classified here and in GP4. These results show that quadruple Galois covers serve as examples and counterexamples to some questions on graded rings and normal generation of linear systems on an algebraic surface.

\section{Notation And CONVEntions}

Convention. We work over an algebraically closed field of characteristic 0 .

Notation 1.1. We will follow these conventions:

(1) Throughout this article, unless otherwise stated, $W$ will be an embedded smooth projective algebraic surface of minimal degree, i.e., whose degree is equal to its codimension in projective space plus 1 .

(2) Throughout this article, unless otherwise stated, $X$ will be a projective algebraic normal surface with at worst canonical singularities (that is, rational double points). We will denote by $\omega_{X}$ the canonical bundle of $X$.

We recall the following standard notation:

(3) By $\mathbf{F}_{e}$ we denote the Hirzebruch surface whose minimal section has selfintersection $-e$. If $e>0$ let $C_{0}$ denote the minimal section of $\mathbf{F}_{e}$ and let $f$ be one of the fibers of $\mathbf{F}_{e}$. If $e=0, C_{0}$ will be a fiber of one of the families of lines and $f$ will be a fiber of the other family of lines. 
(4) If $a, b$ are integers such that $0<a \leq b$, consider two disjoint linear subspaces $\mathbf{P}^{a}$ and $\mathbf{P}^{b}$ of $\mathbf{P}^{a+b+1}$. We denote by $S(a, b)$ the smooth rational normal scroll obtained by joining corresponding points of a rational normal curve in $\mathbf{P}^{a}$ and a rational normal curve of $\mathbf{P}^{b}$. Recall that $S(a, b)$ is the image of $\mathbf{F}_{e}$ by the embedding induced by the complete linear series $\left|C_{0}+m f\right|$, with $a=m-e, b=m$ and $m \geq e+1$.

If $a=b$, the linear series $\left|m C_{0}+f\right|$ also gives a minimal degree embedding of $\mathbf{F}_{0}$, equivalent to the previous one by the automorphism of $\mathbf{P}^{1} \times \mathbf{P}^{1}=\mathbf{F}_{0}$ swapping the factors. In this case our convention will always be to choose $C_{0}$ and $f$ so that, when $W$ is a smooth rational normal scroll, $W$ is embedded by $\left|C_{0}+m f\right|$.

If in addition $m=1, C_{0}$ and $f$ are indistinguisable in both $\mathbf{F}_{0}$ and $S(1,1)$, so, in such a case, for us $C_{0}$ will denote the fiber of any of the families of lines of $\mathbf{F}_{0}$ and $f$ will denote the fiber of the other family.

For details about rational ruled surfaces and rational singularities we refer the reader to $([\mathrm{Ba}])$.

Definition 1.2. Let $X$ and $W$ be as in the previous notation.

a) We will say that a surjective morphism $X \stackrel{\varphi}{\longrightarrow} W$ is a canonical cover of $W$ if $X$ is a surface of general type whose canonical bundle $\omega_{X}$ is ample and base-point-free and $\varphi$ is the canonical morphism of $X$.

b) If $G$ is a finite group acting on $X$ so that $X / G=W$ and $X \stackrel{\varphi}{\longrightarrow} W$ is the projection from $X$ to $X / G$, then we will say that $X \stackrel{\varphi}{\longrightarrow} W$ is a Galois cover with group $G$.

Remark 1.3. If $X \stackrel{\varphi}{\longrightarrow} W$ is a Galois cover, $\varphi$ is flat since $W$ is smooth.

Remark 1.4. Although we have assumed $X$ to have canonical singularities, some results hold in greater generality. Precisely, if for the purpose of this remark we ignore notation 2) above and $X$ is assumed to be a normal, locally Gorenstein surface instead, then Definition 1.2 still makes sense and Theorems 4.1, 5.1, 5.2, 6.1 and 6.2 hold. We can further relax the hypotheses on $X$ in the converse parts of Theorems 4.1, 5.1, 5.2, 6.1 and 6.2, and they hold if $X$ is just assumed to be smooth in codimension 1 , since in that case these covers are Gorenstein.

\section{Some General Results on quAdruple CANONICAL COVERS}

The fact that a cover $X \stackrel{\varphi}{\longrightarrow} W$ is induced by the canonical morphism imposes certain constraints on $\varphi^{*} \mathcal{O}_{X}$. In this section we exploit this to obtain some useful information on the vector bundle structure of $\varphi^{*} \mathcal{O}_{X}$. We get more information if $\varphi^{*} \mathcal{O}_{X}$ splits as a direct sum of line bundles. This is the case for Galois covers, so we will use the results of this section when we study Galois covers in the subsequent sections.

Proposition 2.1. Let $X \stackrel{\varphi}{\longrightarrow} W$ be a quadruple canonical cover of $W$. Let $H=$ $\mathcal{O}_{W}(1)$.

(1) Then $\varphi_{*} \mathcal{O}_{X}$ is a vector bundle on $W$ and

$$
\varphi_{*} \mathcal{O}_{X}=\mathcal{O}_{W} \oplus E \oplus\left(\omega_{W} \otimes H^{*}\right)
$$

with $E$ a vector bundle over $W$ of rank 2. 
(2) If in addition $\varphi_{*} \mathcal{O}_{X}$ splits as a sum of line bundles, then

$$
\varphi_{*} \mathcal{O}_{X}=\mathcal{O}_{W} \oplus L_{1}^{*} \oplus L_{2}^{*} \oplus\left(\omega_{W} \otimes H^{*}\right)
$$

with $L_{1}^{*} \otimes L_{2}^{*}=\omega_{W} \otimes H^{*}$.

Proof. Recall that, by Definition 1.2, $\varphi$ is finite, $W$ is smooth and $X$ is locally Cohen-Macaulay. Then $\varphi$ is flat and hence $\varphi_{*} \mathcal{O}_{X}$ is a vector bundle over $\mathcal{O}_{W}$ of rank 4. Moreover, $\varphi_{*} \mathcal{O}_{X}=\mathcal{O}_{W} \oplus E^{\prime}$, where $E^{\prime}$ is the trace zero module of $\varphi$. From relative duality we have

$$
\varphi_{*} \omega_{X}=\left(\varphi_{*} \mathcal{O}_{X}\right)^{*} \otimes \omega_{W} .
$$

On the other hand, by hypothesis, $\omega_{X}=\varphi^{*} H$, hence, by projection formula,

$$
\varphi_{*} \omega_{X}=\varphi_{*} \mathcal{O}_{X} \otimes H \text {. }
$$

Then

$$
\omega_{W} \oplus\left(\omega_{W} \otimes\left(E^{\prime}\right)^{*}\right)=H \oplus\left(E^{\prime} \otimes H\right) .
$$

Since $\omega_{W}=H$ is not possible, for $W$ a rational surface, $E^{\prime}=E \oplus\left(\omega_{W} \otimes H^{*}\right)$, with $E$ a vector bundle of rank 2 . If $\varphi_{*} \mathcal{O}_{X}$ splits, let $E=L_{1}^{*} \oplus L_{2}^{*}$. Then

$$
\begin{aligned}
\omega_{W} \oplus\left(\omega_{W} \otimes L_{1}\right) \oplus\left(\omega_{W} \otimes L_{2}\right) \oplus H \\
\quad=H \oplus\left(H \otimes L_{1}^{*}\right) \oplus\left(H \otimes L_{2}^{*}\right) \oplus \omega_{W} .
\end{aligned}
$$

Then taking the determinant of both sides of the equality gives $\left(L_{1}^{*} \otimes L_{2}^{*}\right)^{\otimes 2}=$ $\left(\omega_{W} \otimes H^{*}\right)^{\otimes 2}$. Since $W$ is either $\mathbf{P}^{2}$ or a Hirzebruch surface, then $L_{1}^{*} \otimes L_{2}^{*}=$ $\omega_{W} \otimes H^{*}$.

Now we study in more detail the possible splittings of $\varphi_{*} \mathcal{O}_{X}$ depending on what surface $W$ is. We start with this observation about linear $\mathbf{P}^{2}$ :

Proposition 2.2. Let $X \stackrel{\varphi}{\longrightarrow} W$ be a canonical cover. If $W$ is linear $\mathbf{P}^{2}$, then $X$ is regular if and only if $\varphi_{*} \mathcal{O}_{W}$ splits as a direct sum of line bundles.

Proof. By Proposition 2.1 we know that

$$
\varphi_{*} \mathcal{O}_{X}=\mathcal{O}_{W} \oplus E \oplus \omega_{W}(-1) \text {. }
$$

Since $W=\mathbf{P}^{2}$, the intermediate cohomology of line bundles on $W$ vanishes, so by projection formula $H^{1}\left(\varphi^{*} \mathcal{O}_{W}(k)\right)=H^{1}(E(k))$. By Kodaira vanishing and duality $H^{1}\left(\varphi^{*} \mathcal{O}_{W}(k)\right)=0$ except maybe if $k=0,1$. Then $X$ is regular if and only if $H^{1}(E(k))=0$ for all $k$, and by Horrock's Splitting Criterion, this is equivalent to the splitting of $E$. Thus $X$ is regular if and only if $\varphi_{*} \mathcal{O}_{X}$ splits as a direct sum of line bundles.

The following proposition tells how the restriction of $\varphi_{*} \mathcal{O}_{X}$ to a smooth curve in $\left|\omega_{X}\right|$ splits:

Proposition 2.3. Let $W$ be a surface of minimal degree $r$, not necessarily smooth, let $X \stackrel{\varphi}{\longrightarrow} W$ be a canonical cover of degree 4 and let $C$ be a general smooth irreducible curve in $\left|\mathcal{O}_{W}(1)\right|$. If $X$ is regular, then

$$
\left.\left(\varphi_{*} \mathcal{O}_{X}\right)\right|_{C}=\mathcal{O}_{\mathbf{P}^{1}} \oplus \mathcal{O}_{\mathbf{P}^{1}}(-r-1) \oplus \mathcal{O}_{\mathbf{P}^{1}}(-r-1) \oplus \mathcal{O}_{\mathbf{P}^{1}}(-2 r-2) .
$$

Proof. See [GP2, Lemma 2.3] for details. 
Finally we describe more accurately the splitting of $\varphi_{*} \mathcal{O}_{X}$ in the case where $X$ is regular:

Proposition 2.4. Let $X \stackrel{\varphi}{\longrightarrow} W$ be a quadruple canonical cover. If $\varphi_{*} \mathcal{O}_{X}$ splits as a direct sum of line bundles, then:

(1) If $W$ is linear $\mathbf{P}^{2}$, then

$$
\varphi_{*} \mathcal{O}_{X}=\mathcal{O}_{\mathbf{P}^{2}} \oplus \mathcal{O}_{\mathbf{P}^{2}}(-2) \oplus \mathcal{O}_{\mathbf{P}^{2}}(-2) \oplus \mathcal{O}_{\mathbf{P}^{2}}(-4)
$$

(2) $W$ is not the Veronese surface; and

(3) if $W$ is a rational normal scroll and $X$ is regular, then

$$
\begin{aligned}
\varphi_{*} \mathcal{O}_{X}=\mathcal{O}_{W} \oplus \mathcal{O}_{W}\left(-C_{0}-(m+1) f\right) \\
\oplus \mathcal{O}_{W}\left(-2 C_{0}-(e+1) f\right) \oplus \mathcal{O}_{W}\left(-3 C_{0}-(m+e+2) f\right),
\end{aligned}
$$

where $2 m-e$ is the degree of $W$.

Proof. To prove (1) recall that, by Proposition 2.2 $X$ is regular. Then by Proposition 2.3 the restriction of $\varphi_{*} \mathcal{O}_{X}$ to a line of $W$ is

$$
\mathcal{O}_{\mathbf{P}^{1}} \oplus \mathcal{O}_{\mathbf{P}^{1}}(-2) \oplus \mathcal{O}_{\mathbf{P}^{1}}(-2) \oplus \mathcal{O}_{\mathbf{P}^{1}}(-4),
$$

so (1) is clear.

For (2) $W$ is isomorphic to $\mathbf{P}^{2}$ and $\mathcal{O}_{W}(1)=\mathcal{O}_{\mathbf{P}^{2}}(2)$. Then, by Proposition 2.1, $\varphi_{*} \mathcal{O}_{X}=\mathcal{O}_{\mathbf{P}^{2}} \oplus E \oplus \mathcal{O}_{\mathbf{P}^{2}}(-5)$. Let $C$ be a smooth conic in $W$. If $X$ is irregular, then by Proposition 2.2. $\varphi_{*} \mathcal{O}_{X}$ cannot split completely. If $X$ is regular, then according to Proposition 2.3

$$
\left(\varphi_{*} \mathcal{O}_{X}\right)_{C}=\mathcal{O}_{\mathbf{P}^{1}} \oplus \mathcal{O}_{\mathbf{P}^{1}}(-5) \oplus \mathcal{O}_{\mathbf{P}^{1}}(-5) \oplus \mathcal{O}_{\mathbf{P}^{1}}(-10) .
$$

Then if $E=L_{1}^{*} \oplus L_{2}^{*}$ with $L_{1}$ and $L_{2}$ line bundles, $\left.L_{1}^{*}\right|_{C}=\left.L_{2}^{*}\right|_{C}=\mathcal{O}_{\mathbf{P}^{1}}(-5)$, but since $C$ is a conic the degrees of $\left.L_{1}\right|_{C}$ and $\left.L_{2}\right|_{C}$ are even integers, so we get a contradiction.

For $(3)$ recall that $\omega_{W}=\mathcal{O}_{W}\left(-2 C_{0}-(e+2) f\right)$ and that $\mathcal{O}_{W}(1)=\mathcal{O}_{W}\left(C_{0}+m f\right)$. Then it follows by assumption and Proposition 2.1 that

$$
\varphi_{*} \mathcal{O}_{X}=\mathcal{O}_{W} \oplus L_{1}^{*} \oplus L_{2}^{*} \oplus \mathcal{O}_{W}\left(-3 C_{0}-(m+e+2) f\right),
$$

and $L_{1} \otimes L_{2}=\mathcal{O}_{W}\left(3 C_{0}+(m+e+2) f\right)$. Then, if we set $L_{1}=\mathcal{O}_{W}\left(a_{1} C_{0}+b_{1} f\right)$ and $L_{2}=\mathcal{O}_{W}\left(a_{2} C_{0}+b_{2} f\right)$, it follows that

$$
\begin{aligned}
a_{1}+a_{2} & =3, \\
b_{1}+b_{2} & =m+e+2 .
\end{aligned}
$$

We now show that $a_{i} \geq 1$ for $i=1,2$. Since $\varphi$ is induced by the complete linear series of $\varphi^{*} \mathcal{O}_{W}\left(C_{0}+m f\right)$, then $\left(1-a_{i}\right) C_{0}+\left(m-b_{i}\right) f$ is non-effective. Then, if $a_{i} \leq 0$, $b_{i} \geq m+1$. On the other hand, since $X$ is regular and $H^{1}\left(L_{i}^{*}\right) \subset H^{1}\left(\varphi_{*} \mathcal{O}_{X}\right)=0$, it follows that $H^{1}\left(\mathcal{O}_{W}\left(-a_{i} C_{0}-b_{i} f\right)\right)=0$. Then, if $a_{i} \leq 0, b_{i} \leq 1$, then $m \leq 0$, which is impossible, because $m \geq e+1 \geq 1$. Then, since $a_{1}+a_{2}=3, a_{i}$ is either 1 or 2 . Let us set $a_{1}=1$. Then, since $\left(1-a_{1}\right) C_{0}+\left(m-b_{1}\right) f$ cannot be effective, $b_{1} \geq m+1$. On the other hand, $a_{2}=2$, and since $0=h^{1}\left(L_{2}^{*}\right)=$ $h^{1}\left(\mathcal{O}_{W}\left(-2 C_{0}-b_{2} f\right)\right)=h^{1}\left(\mathcal{O}_{W}\left(\left(b_{2}-e-2\right) f\right)\right)=h^{1}\left(\mathcal{O}_{\mathbf{P}^{1}}\left(b_{2}-e-2\right)\right)$, then $b_{2} \geq e+1$. Since $b_{1}+b_{2}=m+e+2$, then $b_{1}=m+1$ and $b_{2}=e+1$.

The purpose of this paper is to study canonical quadruple Galois covers, and we will focus on them in the next sections. Meanwhile, Proposition 2.4 already yields a fact regarding these covers which is worth remarking. Note that a canonical Galois 
cover of the Veronese surface is flat, because the Veronese surface is smooth. Then, being $\varphi$ flat and Galois, $\varphi_{*} \mathcal{O}_{X}$ splits completely, so we have this

Corollary 2.5. There are no quadruple Galois canonical covers of the Veronese surface.

\section{General description of quadruple Galois Canonical covers}

If $X \stackrel{\varphi}{\longrightarrow} W$ is a Galois cover, it is well known that the action on $X$ of the Galois group descomposes $\varphi_{*} \mathcal{O}_{X}$ as a direct sum of eigensheaves. On the other hand $\varphi_{*} \mathcal{O}_{X}$ is a sheaf of $\mathcal{O}_{W}$-algebras, whose multiplicative structure can be described explicitly and is well known (see for instance $[\mathrm{HM}]$ or $\mathrm{Ca}]$ ). In the case of Galois canonical covers of surfaces, Proposition 2.1 (2) gives us some extra information, and the multiplicative structure becomes simpler to state. This is done in Remark 3.1 below; in Corollary 3.2 we translate this data into a geometric description.

Remark 3.1. Let $X \stackrel{\varphi}{\longrightarrow} W$ be a quadruple Galois canonical cover with Galois group $G$ and let

$$
\varphi_{*} \mathcal{O}_{X}=\mathcal{O}_{W} \oplus L_{1}^{*} \oplus L_{2}^{*} \oplus L_{3}^{*}
$$

be the splitting of $\varphi_{*} \mathcal{O}_{X}$ as a sum of line bundles induced by the action of $G$, for which $L_{1} \otimes L_{2}=L_{3}$ (this is possible by Proposition 2.1 (2)). Then

(1) If $G=\mathbf{Z}_{2} \times \mathbf{Z}_{2}$, there exist effective Cartier divisors $D_{1}, D_{2}$ and $D_{3}$ so that the multiplicative structure of $\varphi_{*} \mathcal{O}_{X}$ works as follows:

$$
\begin{array}{lll}
L_{i}^{*} \otimes L_{i}^{*} & \stackrel{D_{j}+D_{k}}{\longrightarrow} & \mathcal{O}_{\mathfrak{Y}}, \\
L_{j}^{*} \otimes L_{k}^{*} & \stackrel{D_{i}}{\longrightarrow} & L_{i}^{*}
\end{array}
$$

and $D_{3}=0$.

(2) If $G=\mathbf{Z}_{4}$, there exist effective Cartier divisors $D_{1}$ and $D_{2}$ on $W$ so that the multiplicative structure of $\varphi_{*} \mathcal{O}_{X}$ works as follows:

$$
\begin{array}{lll}
L_{1}^{*} \otimes L_{1}^{*} & \stackrel{\cdot D_{1}}{\longrightarrow} & L_{2}^{*}, \\
L_{1}^{*} \otimes L_{2}^{*} & \longrightarrow & L_{3}^{*}, \\
L_{1}^{*} \otimes L_{3}^{*} & \stackrel{D_{1}+D_{2}}{\longrightarrow} & \mathcal{O}_{W}, \\
L_{2}^{*} \otimes L_{2}^{*} & \stackrel{D_{2}}{\longrightarrow} & \mathcal{O}_{W}, \\
L_{2}^{*} \otimes L_{3}^{*} & \stackrel{. D_{2}}{\longrightarrow} & L_{1}^{*}, \\
L_{3}^{*} \otimes L_{3}^{*} & \stackrel{D_{1}+D_{2}}{\longrightarrow} & L_{2}^{*} .
\end{array}
$$

Corollary 3.2. Let $X \stackrel{\varphi}{\longrightarrow} W$ be a canonical Galois cover of a smooth surface of minimal degree with Galois group $G$ and keep the notation in Remark 3.1. Then,

(1) if $G=\mathbf{Z}_{2} \times \mathbf{Z}_{2}, X$ is the fiber product over $W$ of two double covers, $X_{1} \stackrel{p_{1}}{\longrightarrow}$ $W$ and $X_{2} \stackrel{p_{2}}{\longrightarrow} W$, branched along $D_{2}$ and $D_{1}$, respectively, and $\varphi$ is the natural map from the fiber product to $W$;

(2) if $G=\mathbf{Z}_{4}$, the cover $X \stackrel{\varphi}{\longrightarrow} W$ is obtained as a composition of two double covers, $X \stackrel{p_{2}}{\longrightarrow} X^{\prime}$ and $X^{\prime} \stackrel{p_{1}}{\longrightarrow} W$, as follows:

a) $p_{1}$ is branched along $D_{2}$;

b) $p_{2}$ is branched along the ramification of $p_{1}$ and along $p^{-1} D_{1}$, and its trace zero module is $p_{1}^{*}\left(L_{1}^{*}\right)$. 
Proof. If $G=\mathbf{Z}_{2} \times \mathbf{Z}_{2}$, then the $\mathcal{O}_{W}$-algebra $p_{*} \mathcal{O}_{X}$ has three subalgebras $\mathcal{O}_{W} \oplus L_{i}^{*}$ corresponding to three double covers $X_{i} \stackrel{p_{i}}{\longrightarrow} W$ for $i=1,2,3$ which are branched along $D_{j}+D_{k}$ where $i \neq j, j \neq k, k \neq i$. Recall (see Remark 3.1) that $L_{1} \otimes L_{2}=L_{3}$ and therefore $D_{3}=0$, hence $X_{1} \stackrel{p_{1}}{\longrightarrow} W$ is branched along $D_{2}$ and $X_{2} \stackrel{p_{2}}{\longrightarrow} W$ is branched along $D_{1}$. Then a local argument shows that the algebra structure of $p_{*} \mathcal{O}_{X}$ described in Remark 3.1 (1) is in fact the tensor product over $\mathcal{O}_{W}$ of the algebras $\mathcal{O}_{W} \oplus L_{1}^{*}$ and $\mathcal{O}_{W} \oplus L_{2}^{*}$.

If $G=\mathbf{Z}_{4}$, then the $\mathcal{O}_{W}$-algebra $p_{*} \mathcal{O}_{X}$ has one proper subalgebra, namely $\mathcal{O}_{W}$ $\oplus L_{2}^{*}$. This induces an intermediate cover $X^{\prime} \stackrel{p_{1}}{\longrightarrow} W$, branched along $D_{2}$. Looking locally at the multiplicative structure described in Remark 3.1 (2) yields the rest of the geometric description of $p_{2}$.

\section{Galois covers of $\mathbf{P}^{2}$}

In this section we deal with the easier case of canonical covers of the projective plane. A priori one could distinguish two cases: either $W$ is linear $\mathbf{P}^{2}$ or $W$ is the Veronese surface. However, as pointed out in Corollary 2.5, there are no canonical quadruple Galois covers of the Veronese surface, so we will only have to study the case of $W$ being linear $\mathbf{P}^{2}$.

Theorem 4.1. Let $W$ be linear $\mathbf{P}^{2}$ and let $X \stackrel{\varphi}{\longrightarrow} W$ be a quadruple Galois canonical cover.

(1) If the Galois group of $\varphi$ is $\mathbf{Z}_{4}$, then $\varphi$ is the composition of two flat double covers $X_{1} \stackrel{p_{1}}{\longrightarrow} W$ and $X \stackrel{p_{2}}{\longrightarrow} X_{1}$; the cover $p_{1}$ is branched along a quartic and the cover $p_{2}$ is branched along the ramification of $p_{1}$ and the pullback by $p_{1}$ of a conic, and its trace zero module is $p_{1}^{*} \mathcal{O}_{\mathbf{P}^{2}}(2)$.

(2) If the Galois group of $\varphi$ is $\mathbf{Z}_{2} \times \mathbf{Z}_{2}$, then $X$ is the fiber product over $W$ of two double covers of linear $\mathbf{P}^{2}$, each of them branched along a quartic, and $\varphi$ is the natural map from the fiber product to $W$.

Conversely, let $X \stackrel{\varphi}{\longrightarrow} W$ be a cover of linear $\mathbf{P}^{2}$.

$\left(1^{\prime}\right)$ If $\varphi$ is the composition of two flat double covers $X_{1} \stackrel{p_{1}}{\longrightarrow} W$ and $X \stackrel{p_{2}}{\longrightarrow} X_{1}$ as described in 1) above, then $\varphi$ is a Galois canonical cover with group $\mathbf{Z}_{4}$.

$\left(2^{\prime}\right)$ If $\varphi$ is the natural map to $W$ from the fiber product over $W$ of two double covers as described in 2) above, then $\varphi$ is a Galois canonical cover with group $\mathbf{Z}_{2} \times \mathbf{Z}_{2}$.

Proof. Corollary 3.2 tells the general structure of $\varphi$, so, to prove (1) and (2) we only need to find out the degrees of the branch divisors. This follows from Proposition 2.4. Now we prove the converse. Clearly, a cover $\varphi$ as in $\left(1^{\prime}\right)$ is Galois with group $\mathbf{Z}_{4}$. Likewise, a cover $\varphi$ as in $\left(2^{\prime}\right)$ is Galois with group $\mathbf{Z}_{2} \times \mathbf{Z}_{2}$. On the other hand, it easily follows from the ramification formula that the canonical of $X$ is $\varphi^{*} \mathcal{O}_{\mathbf{P}^{2}}(1)$, so in particular $X$ is a surface of general type and $\omega_{X}$ is base-point-free. Finally to prove that $\varphi$ is indeed the morphism induced by $H^{0}\left(\omega_{X}\right)$ we see that $H^{0}\left(\varphi^{*} \mathcal{O}_{\mathbf{P}^{2}}(1)\right)=H^{0}\left(\mathcal{O}_{\mathbf{P}^{2}}(1)\right)$. Indeed, a morphism as in $\left.1^{\prime}\right)$ or $\left.2^{\prime}\right)$ satisfies

$$
\varphi_{*} \mathcal{O}_{X}=\mathcal{O}_{\mathbf{P}^{2}} \oplus \mathcal{O}_{\mathbf{P}^{2}}(-2) \oplus \mathcal{O}_{\mathbf{P}^{2}}(-2) \oplus \mathcal{O}_{\mathbf{P}^{2}}(-4),
$$

hence the equality follows from pushing down $\varphi^{*} \mathcal{O}_{\mathbf{P}^{2}}(1)$ to $W$ and computing global sections there. 
We further describe the Galois covers appearing in Theorem 4.1

Corollary 4.2. Let $W$ be linear $\mathbf{P}^{2}$ and let $X \stackrel{\varphi}{\longrightarrow} W$ be a Galois canonical cover of degree 4. If the Galois group of $\varphi$ is $\mathbf{Z}_{4}$, then $X$ is singular and the mildest possible set of singularities on $X$ consists of 8 points of type $A_{1}$.

Proof. Just observe that $X_{1} \stackrel{p_{1}}{\longrightarrow} W$ is branched along a quartic $D_{2}$ of $\mathbf{P}^{2}$ and $X \stackrel{p_{2}}{\longrightarrow} X_{1}$ is branched along the ramification of $p_{1}$ and $p_{1}^{*} D_{1}$, where $D_{1}$ is a conic of $\mathbf{P}^{2}$. If $D_{1}$ and $D_{2}$ are both smooth and meet transversaly, then $X_{1}$ is smooth and the branch locus of $p_{2}$ has 8 singular points of type $A_{1}$, so $X$ is smooth except at 8 points, which are singularities of type $A_{1}$.

We end the section by remarking the existence of examples of covers like those appearing in Theorem 4.1 .

Proposition 4.3. Let $W$ be linear $\mathbf{P}^{2}$.

(1) There exist canonical covers $X \stackrel{\varphi}{\longrightarrow} W$ with Galois group $\mathbf{Z}_{4}$ (that is, covers as in Theorem $4.1(1))$ with 8 singularities of type $A_{1}$ as only singularities.

(2) There exist canonical covers $X \stackrel{\varphi}{\longrightarrow} W$ with Galois group $\mathbf{Z}_{2} \times \mathbf{Z}_{2}$ (that is, covers as in Theorem $4.1(2))$ with $X$ smooth.

Proof. We first deal with (1). By the converse part in Theorem 4.1 and following the notation and arguments of the proof of Corollary 4.2, it suffices to choose a smooth quartic as $D_{1}$ and a smooth conic as $D_{2}$, meeting transversally. This is possible by Bertini. Analogously, for (2) it suffices to pick two smooth quartics, meeting transversally, as branch divisors. Note that, in both cases, one can construct examples of $X$ with worse singularities by allowing $D_{1}+D_{2}$ to have worse singularities.

\section{Bidouble COVERS OF RATIONAL NORMAL SCROLlS}

In the next two sections we proceed to classify quadruple Galois canonical covers of smooth rational normal scrolls. We start by those with Galois group $\mathbf{Z}_{2} \times \mathbf{Z}_{2}$. Having in account Corollary 3.2 we already know that they are the fiber product of two double covers. Thus to complete their description we will find out what are the branch loci of the double covers. We start with the case where $X$ is regular:

Theorem 5.1. Let $W=S(m-e, m)$ be a smooth rational normal scroll. If $X$ is regular and $X \stackrel{\varphi}{\longrightarrow} W$ is a Galois canonical cover with Galois group $\mathbf{Z}_{2} \times \mathbf{Z}_{2}$, then $X$ is the fiber product over $W$ of two double covers of $X_{1} \stackrel{p_{1}}{\longrightarrow} W$ and $X_{2} \stackrel{p_{2}}{\longrightarrow} W$ and $\varphi$ is the natural map from the fiber product to $W$. Let the branch divisors $D_{2}$, $D_{1}$ of $p_{1}, p_{2}$ be linearly equivalent to $2 a_{2} C_{0}+2 b_{2} f$ and $2 a_{1} C_{0}+2 b_{1} f$, respectively. Then $0 \leq e \leq 2, m \geq e+1, a_{1}=1, a_{2}=2, b_{1}=m+1$ and $b_{2}=e+1$.

Conversely, let $W=S(m, m-e)$ be such that $0 \leq e \leq 2$ and $m \geq e+1$ and let $X \stackrel{\varphi}{\longrightarrow} W$ be the natural map to $W$ from the fiber product over $W$ of two flat double covers $p_{1}$ and $p_{2}$ with branch divisors as described above. Then $X$ is regular and $X \stackrel{\varphi}{\longrightarrow} W$ is a Galois canonical cover with Galois group $\mathbf{Z}_{2} \times \mathbf{Z}_{2}$.

Proof. Since $X$ is regular, Proposition 2.4 (3) yields

$$
\begin{aligned}
\varphi_{*} \mathcal{O}_{X}=\mathcal{O}_{W} \oplus \mathcal{O}_{W}\left(-C_{0}-(m+1) f\right) \\
\\
\oplus \mathcal{O}_{W}\left(-2 C_{0}-(e+1) f\right) \oplus \mathcal{O}_{W}\left(-3 C_{0}-(m+e+2) f\right) .
\end{aligned}
$$


Then Remark 3.1 and Corollary 3.2 tell us that $X$ is the fiber product over $W$ of two double covers of $X_{1} \stackrel{p_{1}}{\longrightarrow} W$ and $X_{2} \stackrel{p_{2}}{\longrightarrow} W$ with trace zero modules $L_{1}^{*}=$ $\mathcal{O}_{W}\left(-2 C_{0}-(e+1) f\right)$ and $L_{2}^{*}=\mathcal{O}_{W}\left(-C_{0}-(m+1) f\right)$, respectively, or equivalently, branched along divisors $D_{2} \sim 4 C_{0}+2(e+1) f$ and $D_{1} \sim 2 C_{0}+2(m+1) f$, respectively. Thus $a_{1}=1, a_{2}=2, b_{1}=m+1$ and $b_{2}=e+1$. Recall that $W$ is isomorphic to the Hirzebruch surface $\mathbf{F}_{e}$. Since $W$ is smooth, $m \geq e+1$, hence the only thing left to prove is $e \leq 2$. The covers $p_{1}$ and $p_{2}$ fit in the commutative diagram

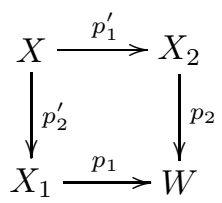

where $p_{1}^{\prime}$ and $p_{2}^{\prime}$ are also double covers. Moreover the branch divisor of $p_{1}^{\prime}$ is $p_{2}^{*} D_{2}$. Suppose that $e \geq 3$. Then, since $D_{2}$ is linearly equivalent to $4 C_{0}+(2 e+2) f, D_{2}$ has $2 C_{0}$ as a fixed component. Then the branch divisor of $p_{1}^{\prime}$ is non-reduced, so $X$ is non-normal and we get a contradiction. Therefore $e=0,1$ or 2 .

To prove the converse assume now that $X \stackrel{\varphi}{\longrightarrow} W$ is the natural map from the fiber product over a smooth scroll $W=S(m, m-e)$ of two double covers $p_{1}$ and $p_{2}$ of $W$, branched, respectively, along divisors $D_{2}$ linearly equivalent to $2 a_{2} C_{0}+2 b_{2} f$ and $D_{1}$ linearly equivalent to $2 a_{1} C_{0}+2 b_{1} f$. Assume in addition that $0 \leq e \leq 2$, $a_{1}=1, a_{2}=2, b_{1}=m+1$ and $b_{2}=e+1$. Then it is clear that $\varphi$ is a Galois cover with Galois group $\mathbf{Z}_{2} \times \mathbf{Z}_{2}$ and

$$
\begin{aligned}
\varphi_{*} \mathcal{O}_{X}=\mathcal{O}_{W} \oplus \mathcal{O}_{W}\left(-a_{1} C_{0}-b_{1} f\right) \\
\\
\oplus \mathcal{O}_{W}\left(-a_{2} C_{0}-b_{2} f\right) \oplus \mathcal{O}_{W}\left(-\left(a_{1}+a_{2}\right) C_{0}-\left(b_{1}+b_{2}\right) f\right) .
\end{aligned}
$$

A standard computation shows that none of the four direct summands of $p_{*} \mathcal{O}_{X}$ have intermediate cohomology, hence $H^{1}\left(\mathcal{O}_{X}\right)=0$. On the other hand if $L_{2}=$ $\mathcal{O}_{W}\left(a_{1} C_{0}+b_{1} f\right)$ and $L_{1}=\mathcal{O}_{W}\left(a_{2} C_{0}+b_{2} f\right)$, then $L_{1} \otimes L_{2}=\mathcal{O}_{W}\left(3 C_{0}+(m+e+2) f\right)=$ $\omega_{W}^{*}(1)$. Then, by the ramification formula $\omega_{X}=\varphi^{*} \mathcal{O}_{W}(1)$ so $X$ is a surface of general type whose canonical bundle is base-point-free. The only thing left to be shown is that $X \stackrel{\varphi}{\longrightarrow} W$ is the canonical morphism of $X$. For that it is enough to see that $H^{0}\left(\mathcal{O}_{W}(1)\right)=H^{0}\left(\omega_{X}\right)$. But

$$
\begin{aligned}
H^{0}\left(\omega_{X}\right)= & H^{0}\left(\varphi^{*} \mathcal{O}_{W}\left(C_{0}+m f\right)\right) \\
= & H^{0}\left(\mathcal{O}_{W}\left(C_{0}+m f\right)\right) \oplus H^{0}\left(\mathcal{O}_{W}\left(\left(1-a_{1}\right) C_{0}+\left(m-b_{1}\right) f\right)\right) \\
& \oplus H^{0}\left(\mathcal{O}_{W}\left(\left(1-a_{2}\right) C_{0}+\left(m-b_{2}\right) f\right)\right) \\
& \oplus H^{0}\left(\mathcal{O}_{W}\left(1-a_{1}-a_{2}\right) C_{0}+\left(m-b_{1}-b_{2}\right) f\right) .
\end{aligned}
$$

Now, because of the restrictions on $a_{1}, a_{2}, b_{1}$ and $b_{2}, H^{0}\left(\mathcal{O}_{W}\left(\left(1-a_{1}\right) C_{0}+\left(m-b_{1}\right) f\right)\right)$, $H^{0}\left(\mathcal{O}_{W}\left(\left(1-a_{2}\right) C_{0}+\left(m-b_{2}\right) f\right)\right)$ and $H^{0}\left(\mathcal{O}_{W}\left(1-a_{1}-a_{2}\right) C_{0}+\left(m-b_{1}-b_{2}\right) f\right)$ vanish.

Now we go on to classify Galois quadruple covers with group $\mathbf{Z}_{2} \times \mathbf{Z}_{2}$ when $X$ is irregular:

Theorem 5.2. Let $W$ be a smooth rational normal scroll $S(m-e, m)$. If $X$ is irregular and $X \stackrel{\varphi}{\longrightarrow} W$ is a Galois canonical cover with Galois group $\mathbf{Z}_{2} \times \mathbf{Z}_{2}$, then $X$ is the fiber product over $W$ of two double covers of $X_{1} \stackrel{p_{1}}{\longrightarrow} W$ and $X_{2} \stackrel{p_{2}}{\longrightarrow} W$ 
and $\varphi$ is the natural map from the fiber product to $W$. Let the branch divisors $D_{2}$, $D_{1}$ of $p_{1}, p_{2}$ be linearly equivalent to $2 a_{2} C_{0}+2 b_{2} f$ and $2 a_{1} C_{0}+2 b_{1} f$, respectively. Then $e=0, m \geq 1$ and one of the following happens:

(1) $a_{1}=0, a_{2}=3, b_{1}=m+1, b_{2}=1$.

(2) $a_{1}=0, a_{2}=3, b_{1}=m+2, b_{2}=0$.

(3) $a_{1}=1, a_{2}=2, b_{1}=m+2, b_{2}=0$.

In addition, in case (1), $q(X)=m$; in case (2), $q(X)=m+3$; and in case (3), $q(X)=1$.

Conversely, let $X \stackrel{\varphi}{\longrightarrow} W$ be the natural map to $W=S(m, m)$ from the fiber product over $W$ of two flat double covers $p_{1}$ and $p_{2}$ with branch divisors satisfying (1), (2) or (3) above. Then $X$ is irregular and $X \stackrel{\varphi}{\longrightarrow} W$ is a Galois canonical cover with Galois group $\mathbf{Z}_{2} \times \mathbf{Z}_{2}$.

Proof. From Proposition 2.1 and Corollary 3.2 it follows that $X$ is the fiber product of two double covers branched along divisors

$$
D_{2} \sim 2\left(a_{2} C_{0}+b_{2} f\right) \text { and } D_{1} \sim 2\left(a_{1} C_{0}+b_{1} f\right),
$$

respectively, that

$$
\begin{aligned}
\varphi_{*} \mathcal{O}_{X}=\mathcal{O}_{W} & \oplus \mathcal{O}_{W}\left(-a_{1} C_{0}-b_{1} f\right) \\
& \oplus \mathcal{O}_{W}\left(-a_{2} C_{0}-b_{2} f\right) \oplus \mathcal{O}_{W}\left(-\left(a_{1}+a_{2}\right) C_{0}-\left(b_{1}+b_{2}\right) f\right)
\end{aligned}
$$

and $\omega_{W}(-1)=\mathcal{O}_{W}\left(-\left(a_{1}+a_{2}\right) C_{0}-\left(b_{1}+b_{2}\right) f\right)$. Since $\omega_{W}=\mathcal{O}_{W}\left(-2 C_{0}-(e+2) f\right)$, we obtain

$$
\begin{aligned}
a_{1}+a_{2} & =3 \\
b_{1}+b_{2} & =m+e+2 .
\end{aligned}
$$

On the other hand since $D_{i}$ is effective and linearly equivalent to $2\left(a_{i} C_{0}+b_{i} f\right)$, then $a_{i}, b_{i} \geq 0$. We set $a_{1}=0,1$ (in which case, $a_{2}=3,2$ ). Since $\varphi$ is induced by the complete linear series of $\varphi^{*} \mathcal{O}_{W}\left(C_{0}+m f\right)$, then $H^{0}\left(\mathcal{O}_{W}\left(\left(1-a_{1}\right) C_{0}+\left(m-b_{1}\right) f\right)\right)=0$, hence $b_{1} \geq m+1$, and from (5.2.1), $b_{2} \leq e+1$. Since both $b_{1}$ and $b_{2}$ are non-negative, $m+1 \leq b_{1} \leq m+e+2$ and $0 \leq b_{2} \leq e+1$.

Now assume $a_{1}=0$. Then $D_{2}$ is linearly equivalent to $6 C_{0}+2 b_{2} f$. Assume also that $e \geq 1$. Then $2 C_{0}$ is a fixed component of $D_{2}$, therefore by the argument made in the proof of Theorem 5.1, $X$ would be non-normal, hence, if $a_{1}=0$, then $e=0$. Then, we have two possibilities: first, $b_{1}=m+1$ and $b_{2}=1$, and second, $b_{1}=m+2$ and $b_{2}=0$. In the first case, $q(X)=m$. In the second case, $q(X)=m+3$.

Now assume $a_{1}=1$. Then $D_{2}$ is linearly equivalent to $4 C_{0}+2 b_{2} f$, and as we argued in the proof of Theorem 5.1, if $e \geq 3, X$ would be non-normal, hence $0 \leq e \leq 2$. Moreover, if $b_{2}<\frac{3 e}{2}, D_{2}$ would have $2 C_{0}$ as a fixed component and $X$ would be non-normal, hence $b_{2} \geq \frac{3 e}{2}$. Recall also that $b_{2} \leq e+1$. Let us now assume that $b_{2}=e+1$. Then $b_{1}=m+1$ and in that case

$$
\begin{aligned}
\varphi_{*} \mathcal{O}_{X}= & \mathcal{O}_{W} \oplus \mathcal{O}_{W}\left(-C_{0}-(m+1) f\right) \\
& \oplus \mathcal{O}_{W}\left(-2 C_{0}-(e+1) f\right) \oplus \mathcal{O}_{W}\left(-3 C_{0}-(m+e+2) f\right) .
\end{aligned}
$$

But then $H^{1}\left(\mathcal{O}_{W}\right), H^{1}\left(\mathcal{O}_{W}\left(-C_{0}-(m+1) f\right)\right), H^{1}\left(\mathcal{O}_{W}\left(-2 C_{0}-(e+1) f\right)\right)$ and $H^{1}\left(\mathcal{O}_{W}\left(-3 C_{0}-(m+e+2) f\right)\right)$ all vanish, hence $X$ would be regular. Therefore $\frac{3 e}{2} \leq b_{2} \leq e$. This implies that $e=0$ and $b_{2}=0$, in which case $b_{1}=m+2$. Then $q(X)=1$. With this we prove that $W=S(m, m)$ and that the only possibilities for the $a_{i} \mathrm{~s}, b_{i}$ s are (1), (2) and (3). 
To prove the converse, assume now that $X \stackrel{\varphi}{\longrightarrow} W$ is the fiber product over $W$ of two double covers $p_{1}$ and $p_{2}$ of $W$, branched, respectively, along divisors $D_{2}$ linearly equivalent to $2 a_{2} C_{0}+2 b_{2} f$ and $D_{1}$ linearly equivalent to $2 a_{1} C_{0}+2 b_{1} f$ satisfying one of the cases (1), (2) or (3). Then it is clear that $\varphi$ is a Galois cover with Galois group $\mathbf{Z}_{2} \times \mathbf{Z}_{2}$, and

$$
\begin{aligned}
\varphi_{*} \mathcal{O}_{X}= & \mathcal{O}_{W} \oplus \mathcal{O}_{W}\left(-a_{1} C_{0}-b_{1} f\right) \\
& \oplus \mathcal{O}_{W}\left(-a_{2} C_{0}-b_{2} f\right) \oplus \mathcal{O}_{W}\left(-\left(a_{1}+a_{2}\right) C_{0}-\left(b_{1}+b_{2}\right) f\right)
\end{aligned}
$$

with $a_{1}, a_{2}, b_{1}, b_{2}$ satisfying (1), (2) or (3). Computing the cohomology of the four direct summands of $\varphi_{*} \mathcal{O}_{X}$ in each case shows that $X$ is always irregular. On the other hand, $\omega_{X}=\varphi^{*} \mathcal{O}_{W}(1)$ by the ramification formula and, in particular, $\omega_{X}$ is base-point-free and $X$ is a surface of general type. Finally one easily sees that $X \stackrel{\varphi}{\longrightarrow} W$ is the canonical morphism of $X$ by showing that $H^{0}\left(\mathcal{O}_{W}(1)\right)=H^{0}\left(\omega_{X}\right)$ as in the proof of Theorem 5.1

Remark 5.3. The description of $D_{1}$ and $D_{2}$ yields that, in case (2) of Theorem 5.2 , $X$ is actually the product of a curve of genus $m+1$ and a curve of genus 3 . This type of surface appears in the examples constructed by Beauville in Be. In the cases described in Theorem 5.2 (1), Theorem 5.2 (3) and Theorem 6.2, there is also a simpler description for $X$ : it can be seen as a double cover, branched along a suitable divisor, of the product of $C \times \mathbf{P}^{1}$, where $C$ is a curve of genus $m$ or 1 , respectively.

We end the section showing the existence of canonical covers such as the ones classified in Theorems 5.1, and 5.2

Proposition 5.4. There exist families of canonical Galois quadruple covers $X \stackrel{\varphi}{\longrightarrow}$ $W$ as in Theorem 5.1 and Theorem 5.2 (1), (2) and (3) with $X$ smooth.

Proof. Families satisfying Theorem 5.1 for $W$ isomorphic to $\mathbf{F}_{0}$ and $\mathbf{F}_{1}$ have been constructed in [GP2, Examples 3.4 and 3.5] (see also [Pe for an example of a bidouble cover of $\mathbf{P}^{1} \times \mathbf{P}^{1}$ ). To construct the remaining examples we argue as in Proposition 4.3. By the converse part of Theorems 5.1] and 5.2 we only need to construct the fiber product of two double covers $X_{1} \stackrel{p_{1}}{\longrightarrow} W$ and $X_{2} \stackrel{p_{2}}{\longrightarrow} W$ branched along suitable divisors $D_{2}$ and $D_{1}$ satisfying the conditions in the statement of Theorems 5.1 and 5.2. Precisely if we choose $D_{1}$ and $D_{2}$ smooth and meeting transversally, $X$ will be smooth. This can be achieved using Bertini once we study how the divisors $D_{1}$ and $D_{2}$ are in each case. Indeed, if we are in the situation of Theorem 5.1] when $W$ is isomorphic to $\mathbf{F}_{2}$, then $D_{1} \sim 2 C_{0}+2(m+1) f$ is very ample and $D_{2}$, since it is linearly equivalent to $4 C_{0}+6 f$, is of the form $C_{0}+D_{2}^{\prime}$, with $D_{2}^{\prime} \cdot C_{0}=0$ and $D_{2}^{\prime}$ base-point-free.

In the cases of Theorem 5.2 (1), (2) and (3) recall that $W$ is isomorphic to $\mathbf{F}_{0}$. In the case of Theorem $5.2(1) D_{1} \sim 2(m+1) f$, hence it can be chosen as the union of $2(m+1)$ distinct lines in one of the two fibrations of $\mathbf{F}_{0}$, and $D_{2}$ is linearly equivalent to $6 C_{0}+2 f$, which is very ample.

In the case of Theorem $5.2(3), D_{1} \sim 2 C_{0}+2(m+2) f$ is very ample and $D_{2} \sim 4 C_{0}$ can be chosen as the union of 4 distinct lines of one of the fibrations of $\mathbf{F}_{0}$. Finally, in the case of Theorem $5.2(2), D_{1}$ and $D_{2}$ can both be taken as the union of distinct lines. Note that one can construct $X$ having singularities if $D_{1}+D_{2}$ is allowed to have worse singularities. 


\section{CyClic COVERS OF RATiOnAL NORMAL SCROLlS}

In this section we study canonical Galois covers, with group $\mathbf{Z}_{4}$, of rational normal scrolls. We again split the cases $X$ regular and $X$ irregular. One of the facts we prove is that these covers are never simple cyclic, as in the case of $\mathbf{P}^{2}$. As we will see in Section 7, there are deeper reasons for this. Another interesting fact we see is that these covers are always singular, having at best singularities of type $A_{1}$.

Theorem 6.1. Let $X \stackrel{\varphi}{\longrightarrow} W$ be a Galois canonical cover of degree 4 and Galois group $\mathbf{Z}_{4}$ with $X$ regular and let $W=S(m-e, m)$ be a smooth rational normal scroll. Then $\varphi$ is the composition of two flat double covers $X_{1} \stackrel{p_{1}}{\longrightarrow} W$ and $X \stackrel{p_{2}}{\longrightarrow} X_{1}$ which are as follows:

(1) The cover $p_{1}$ is branched along a divisor $D_{2}$ on $W$.

(2) The cover $p_{2}$ is branched along the ramification of $p_{1}$ and $p_{1}^{*} D_{1}$ and has trace zero module $p_{1}^{*} \mathcal{O}_{Y}\left(-\frac{1}{2} D_{1}-\frac{1}{4} D_{2}\right)$, where $D_{1}$ is a divisor on $W$.

(3) The scroll $W=S(m-e, m)$, with $0 \leq e \leq 2$ and $m \geq e+1$, and the divisors $D_{1} \sim(2 m-e+1) f, D_{2} \sim 4 C_{0}+(2 e+2) f$.

Conversely, let $X \stackrel{\varphi}{\longrightarrow} W$ be the composition of two flat double covers $X_{1} \stackrel{p_{1}}{\longrightarrow} W$ and $X \stackrel{p_{2}}{\longrightarrow} X_{1}$ as described above; then $\varphi$ is a Galois canonical cover of $W$ with Galois group $\mathbf{Z}_{4}$ and $X$ is regular.

Proof. Corollary 3.2 says that $\varphi$ is the composition of two double covers $X_{1} \stackrel{p_{1}}{\longrightarrow} W$, branched along a divisor $D_{2}$, and $X \stackrel{p_{2}}{\longrightarrow} X_{1}$, branched along the ramification of $p_{1}$ and $p_{1}^{*} D_{1}$, and, according to Remark 3.1 .

$$
\varphi_{*} \mathcal{O}_{X}=\mathcal{O}_{W} \oplus L_{1}^{*} \oplus L_{2}^{*} \oplus L_{3}^{*}
$$

where $L_{1} \otimes L_{2}=L_{3}$. Moreover,

$$
L_{3}=\omega_{W}^{*}(1), L_{1}^{\otimes 2}=L_{2} \otimes \mathcal{O}_{W}\left(D_{1}\right), L_{2}^{\otimes 2}=\mathcal{O}_{W}\left(D_{2}\right),
$$

$p_{1}^{*} L_{1}^{*}$ is the trace zero module of $p_{2}$ and $L_{2}^{*}$ is the trace zero module of $p_{1}$. From this we obtain that the trace zero module of $p_{2}$ is $p_{1}^{*} L_{1}^{*}=p_{1}^{*} \mathcal{O}_{Y}\left(-\frac{1}{2} D_{1}-\frac{1}{4} D_{2}\right)$.

Now we show that $W, D_{1}$ and $D_{2}$ satisfy (3). Recall that $W$ is isomorphic to $\mathbf{F}_{e}$. Since $X$ is regular we can apply Proposition 2.4 (3). Then, since $L_{3}=\omega_{W}^{*}(1)$ we have either

$$
\begin{gathered}
L_{1}^{*}=\mathcal{O}_{W}\left(-C_{0}-(m+1) f\right), L_{2}^{*}=\mathcal{O}_{W}\left(-2 C_{0}-(e+1) f\right) \\
\quad \text { and } L_{3}^{*}=\mathcal{O}_{W}\left(-3 C_{0}-(m+e+2) f\right)
\end{gathered}
$$

or

$$
\begin{gathered}
L_{2}^{*}=\mathcal{O}_{W}\left(-C_{0}-(m+1) f\right), L_{1}^{*}=\mathcal{O}_{W}\left(-2 C_{0}-(e+1) f\right) \\
\quad \text { and } L_{3}^{*}=\mathcal{O}_{W}\left(-3 C_{0}-(m+e+2) f\right) .
\end{gathered}
$$

Case 1: $L_{1}^{*}=\mathcal{O}_{W}\left(-C_{0}-(m+1) f\right), L_{2}^{*}=\mathcal{O}_{W}\left(-2 C_{0}-(e+1) f\right)$. From the previous description, $\varphi$ is the composition of $X_{1} \stackrel{p_{1}}{\longrightarrow} W$, where $p_{1}$ is a double cover branched along a divisor $D_{2}$ linearly equivalent to $4 C_{0}+(2 e+2) f$, and $X \stackrel{p_{2}}{\longrightarrow} X_{1}$, where $p_{2}$ is a double cover branched along the ramification of $p_{1}$ and $p_{1}^{*} D_{1}$, where $D_{1}$ is linearly equivalent to $(2 m-e+1) f$. Recall that $X$ is normal, hence the components of $D_{1}+D_{2}$ have multiplicity 1 and in particular, the fixed part of $\left|4 C_{0}+(2 e+2) f\right|$ contains $C_{0}$ with multiplicity at most 1 . Thus $e \leq 2$. 
Case 2: $L_{2}^{*}=\mathcal{O}_{W}\left(-C_{0}-(m+1) f\right), L_{1}^{*}=\mathcal{O}_{W}\left(-2 C_{0}-(e+1) f\right)$. Again from the description above, $\varphi$ is the composition of $X_{1} \stackrel{p_{1}}{\longrightarrow} W$, where $p_{1}$ is a double cover branched along a divisor $D_{2}$ linearly equivalent to $2 C_{0}+(2 m+2) f$, and $X \stackrel{p_{2}}{\longrightarrow} X_{1}$, where $p_{2}$ is a double cover branched along the ramification of $p_{1}$ and $p_{1}^{*} D_{1}$, where $D_{1}$ is linearly equivalent to $3 C_{0}+(2 e-m+1) f$. Recall that $X$ is normal, hence the components of $D_{1}+D_{2}$ have multiplicity 1 and, in particular, the fixed part of $\left|3 C_{0}+(2 e-m+1) f\right|$ contains $C_{0}$ with multiplicity at most 1 . Thus $m \leq 1$, and in fact $m=1$ and $e=0$. So finally, $W=\mathbf{F}_{0}, D_{1}$ is linearly equivalent to $3 C_{0}$ and $D_{2}$ is linearly equivalent to $2 C_{0}+4 f$. After interchanging $C_{0}$ and $f$ we see that $D_{1}$ and $D_{2}$ satisfy (3) when we set $e=0$ and $m=1$.

Now we prove the converse. It is clear that the morphism $\varphi$ is a Galois cover with group $\mathbf{Z}_{4}$. The ramification formula tells us that

$$
\omega_{X}=\varphi^{*}\left(\omega_{W} \otimes \mathcal{O}_{W}\left(3 C_{0}+(m+e+2) f\right)\right)=\varphi^{*} \mathcal{O}_{W}(1),
$$

so $X$ is a surface of general type with a base-point-free canonical bundle. It is also clear, in both (1) and (2), that

$$
\begin{aligned}
\varphi_{*} \mathcal{O}_{X}=\mathcal{O}_{W} & \oplus \mathcal{O}_{W}\left(-C_{0}-(m+1) f\right) \\
& \oplus \mathcal{O}_{W}\left(-2 C_{0}-(e+1) f\right) \oplus \mathcal{O}_{W}\left(-3 C_{0}-(m+e+2) f\right) .
\end{aligned}
$$

Then to see that $\varphi$ is the canonical morphism of $X$ we compare $H^{0}\left(\omega_{X}\right)=$ $H^{0}\left(\varphi^{*} \mathcal{O}_{W}(1)\right)$ and $H^{0}\left(\mathcal{O}_{W}(1)\right)$. The group $H^{0}\left(\varphi^{*} \mathcal{O}_{W}(1)\right)$ can be computed pushing $\varphi^{*} \mathcal{O}_{W}(1)$ down to $W$ and using (6.1.1), and one sees at once that $H^{0}\left(\omega_{X}\right)=$ $H^{0}\left(\mathcal{O}_{W}(1)\right)$. Finally we see that $H^{1}\left(\mathcal{O}_{X}\right)=0$ also by pushing down to $W$ and using (6.1.1).

Theorem 6.2. Let $X \stackrel{\varphi}{\longrightarrow} W$ be a canonical Galois cover of degree 4 and Galois group $\mathbf{Z}_{4}$ with $X$ irregular and let $W=S(m-e, m)$ be a smooth rational normal scroll. Then the irregularity of $X$ is $q(X)=1$ and $W$ is isomorphic to $\mathbf{F}_{0}$. Moreover, $\varphi$ is the composition of two flat double covers $X_{1} \stackrel{p_{1}}{\longrightarrow} W$ and $X \stackrel{p_{2}}{\longrightarrow} X_{1}$ which are as follows:

(1) The cover $p_{1}$ is branched along a divisor $D_{2}$ on $W$.

(2) The cover $p_{2}$ is branched along the ramification of $p_{1}$ and $p_{1}^{*} D_{1}$ and has trace zero module $p_{1}^{*} \mathcal{O}_{Y}\left(-\frac{1}{2} D_{1}-\frac{1}{4} D_{2}\right)$, where $D_{1}$ is a divisor on $W$.

(3) The scroll $W=S(m, m)$, with $m \geq 1$, and the divisors $D_{1} \sim(2 m+4) f$ and $D_{2} \sim 4 C_{0}$.

Conversely, if $X \stackrel{\varphi}{\longrightarrow} W$ is the composition of two double covers $X_{1} \stackrel{p_{1}}{\longrightarrow} W$ and $X \stackrel{p_{2}}{\longrightarrow} X_{1}$ as described above, then $\varphi$ is a canonical Galois cover of $W$ with Galois group $\mathbf{Z}_{4}$ and $X$ is irregular.

Proof. Using Remark 3.1 and Corollary 3.2 as in the proof of Theorem 6.1 we conclude that $\varphi$ is the composition of two double covers. The first one is $X_{1} \stackrel{p_{1}}{\longrightarrow} W$, is branched along a divisor $D_{2}$ and has trace zero module $L_{2}^{*}$. The second cover is $X \stackrel{p_{2}}{\longrightarrow} X_{1}$, is branched along the ramification of $p_{1}$ and $p_{1}^{*} D_{1}$ and has trace zero module $p_{1}^{*} L_{1}$. Then $L_{1}^{\otimes 2}=L_{2} \otimes \mathcal{O}_{W}\left(D_{1}\right), L_{2}^{\otimes 2}=\mathcal{O}_{W}\left(D_{2}\right)$ and moreover, $L_{1} \otimes L_{2}=L_{3}=\omega_{W}^{*}(1)$. Recall that $W=\mathbf{F}_{e}$ and let $L_{1}=\mathcal{O}_{W}\left(a_{1} C_{0}+b_{1} f\right)$ and 
$L_{2}=\mathcal{O}_{W}\left(a_{2} C_{0}+b_{2} f\right)$. Then we have

$$
\begin{aligned}
a_{1}+a_{2} & =3 \\
b_{1}+b_{2} & =m+e+2 .
\end{aligned}
$$

Since $L_{1}^{\otimes 2} \otimes L_{2}^{*}=\mathcal{O}_{W}\left(D_{1}\right)$ and $L_{2}^{\otimes 2}=\mathcal{O}_{W}\left(D_{2}\right)$ are effective, then $a_{2} \leq 2 a_{1}$, $b_{2} \leq 2 b_{1}$ and $a_{2}, b_{2} \geq 0$. Then $a_{1}, b_{1} \geq 0$ also. Moreover, $a_{1}, b_{1} \geq 1$, otherwise we will contradict $a_{1}+a_{2}=3$ or $b_{1}+b_{2}=m+e+2$. We see that $a_{1}$ cannot be 2 . If $a_{1}=2$, then $D_{1} \sim 3 C_{0}+\left(2 b_{1}-b_{2}\right) f, D_{2} \sim 2 C_{0}+2 b_{2} f, L_{1}=\mathcal{O}_{W}\left(2 C_{0}+b_{1} f\right)$ and $L_{2}=\mathcal{O}_{W}\left(C_{0}+b_{2} f\right)$. Since $X$ is irregular, and $H^{1}\left(\mathcal{O}_{W}\right)=H^{1}\left(L_{2}^{*}\right)=H^{1}\left(L_{3}^{*}\right)=0$, then $H^{1}\left(L_{1}^{*}\right) \neq 0$. This implies $b_{1} \leq e$. Then $b_{1}+b_{2}=m+e+2$ implies $b_{2} \geq m+2$. On the other hand, since $X$ is normal, $C_{0}$ has at most multiplicity 1 in the fixed part of $\left|3 C_{0}+\left(2 b_{1}-b_{2}\right) f\right|$, and this implies $2 b_{1}-b_{2}-2 e \geq 0$. Then we have $2 e-(m+2)-2 e \geq 0$, which is a contradiction. Then the only possibilities are $a_{1}=1$ or $a_{1}=3$.

Case 1: $a_{1}=1$. Then $a_{2}=2$ and $X$ irregular implies $b_{2} \leq e$, since $L_{2}^{*}$ has to be special. The fact that $X$ is normal implies $-3 e+2 b_{2} \geq 0$, since $D_{2}$ cannot have $2 C_{0}$ as a fixed component. Then $b_{2}=e=0$, and, summarizing, $e=0, a_{1}=1, a_{2}=$ $2, b_{1}=m+2$, and $b_{2}=0$. This implies $D_{1} \sim(2 m+4) f$ and $D_{2} \sim 4 C_{0}$.

Case 2: $a_{1}=3$. Then $D_{1} \sim 6 C_{0}+\left(2 b_{1}-b_{2}\right) f$, and since $X$ is normal, $C_{0}$ has at most multiplicity 1 in the fixed part of $\left|D_{1}\right|$, hence $2 b_{1}-b_{2}-5 e \geq 0$. Now since $H^{0}\left(\varphi^{*} \mathcal{O}_{W}\left(C_{0}+m f\right)\right)=H^{0}\left(\mathcal{O}_{W}\left(C_{0}+m f\right)\right)$, we have that $b_{2}>m$, hence $b_{1}<e+2$. Then we get $2 e+4-m-5 e=-3 e-m+4>0$. But $m \geq e+1$, so this gives $-4 e+3>0$, hence $e=0$. In this case, $b_{1}=1$ and $m \geq 1$. Then $b_{2}=m+1 \geq 2$, and since $D_{1}$ is effective, $b_{2}=2$ and $m=1$. Summarizing, $e=0, m=1, a_{1}=3$, $a_{2}=0, b_{1}=1$ and $b_{2}=2$. Then $D_{1} \sim 6 C_{0}$ and $D_{2} \sim 4 f$. After interchanging $C_{0}$ and $f$ we see that $D_{1}$ and $D_{2}$ satisfy (3) when we set $m=1$.

Finally the irregularity of $X$ is $h^{1}\left(\mathcal{O}_{X}\right)=h^{1}\left(\varphi_{*} \mathcal{O}_{X}\right)$. We observe that the computation of $a_{1}, a_{2}, b_{1}$ and $b_{2}$ yields that $\varphi_{*} \mathcal{O}_{X}$ is

$\mathcal{O}_{W} \oplus \mathcal{O}_{W}\left(-C_{0}-(m+2) f\right) \oplus \mathcal{O}_{W}\left(-2 C_{0}\right) \oplus \mathcal{O}_{W}\left(-3 C_{0}-(m+2) f\right)$ or

$$
\mathcal{O}_{W} \oplus \mathcal{O}_{W}\left(-3 C_{0}-f\right) \oplus \mathcal{O}_{W}(-2 f) \oplus \mathcal{O}_{W}\left(-3 C_{0}-3 f\right)
$$

so $h^{1}\left(\mathcal{O}_{X}\right)=h^{1}\left(\mathcal{O}_{W}\left(-2 C_{0}\right)\right)$ or $h^{1}\left(\mathcal{O}_{X}\right)=h^{1}\left(\mathcal{O}_{W}(-2 f)\right)$, and in both cases, are equal to 1 .

We now prove the converse. It is clear that $\varphi$ is Galois with Galois group $\mathbf{Z}_{4}$. Now if $L_{2}^{*}$ is the trace zero module of $p_{1}$ and $p_{1}^{*} L_{1}^{*}$ is the trace zero module of $p_{2}$, then $L_{1} \otimes L_{2}=\mathcal{O}_{W}\left(\frac{1}{2} D_{1}+\frac{3}{4} D_{2}\right)$. Then if $D_{1}$ and $D_{2}$ are as in (1) or (2), $L_{1} \otimes L_{2}=\omega_{W}^{*}(1)$. Then the ramification formula also implies that $\omega_{X}=\varphi^{*} \mathcal{O}_{W}(1)$, therefore $X$ is a surface of general type with a base-point-free canonical bundle. Finally it is also clear that $\varphi_{*} \mathcal{O}_{X}$ is as in (6.2.1), so arguing as in the end of the proof of Theorem 6.1 we see that $\varphi$ is the canonical morphism and $X$ is irregular.

Corollary 6.3. Let $W$ be a smooth rational scroll of degree $r$ and let $X \stackrel{\varphi}{\longrightarrow} W$ be a Galois canonical cover with Galois group $\mathbf{Z}_{4}$ (i.e., a cover like the ones classified in Theorems 6.1 and 6.2). Then $X$ is singular. Moreover,

(1) if $X$ is regular, then the mildest possible set of singularities on $X$ consists of $4(r+1)$ singular points of type $A_{1}$ and

(2) if $X$ is irregular the singularities of $X$ are exactly $4(r+4)$ points of type $A_{1}$. 
Proof. The proof is similar to the proof of Corollary 4.2. In this occasion, $D_{1} \cdot D_{2}=$ $4(r+1)$ if $X$ is regular (see Theorem 6.1 (1) and (2)) and $D_{1} \cdot D_{2}=4(r+4)$ if $X$ is irregular (see Theorem 6.2 (1) and (2)). The surface $X$ has the mildest possible set of singularities if $X_{1}$ is smooth and the branch locus of $X \stackrel{p_{2}}{\longrightarrow} X_{1}$ has the mildest possible set of singularities. This happens if $D_{1}$ and $D_{2}$ are smooth and meet tranversally. In this case the branch locus of $p_{2}$ has only singularities of type $A_{1}$, and so does $X$. Now, if $X$ is irregular, Theorem 6.2 together with the fact that $X$ is normal implies that $D_{1}$ is a union of distinct lines of one of the fibrations of $\mathbf{P}^{1} \times \mathbf{P}^{1}$ and $D_{2}$ is a union of distinct lines of the other fibration, so $D_{1}$ and $D_{2}$ are smooth and meet transversally in any case.

Proposition 6.4. There exist families of quadruple Galois canonical covers as in Theorems 6.1 and 6.2 which have singularities as mild as possible (see Corollary 6.3).

Proof. According to the converse part in Theorems 6.1 and 6.2 we just have to construct a composition of double covers $X \stackrel{p_{2}}{\longrightarrow} X_{1}$ and $X_{1} \stackrel{p_{1}}{\longrightarrow} W$ branched along suitable divisors. Let $D_{1}$ and $D_{2}$ be as in Theorems 6.1 and 6.2. Using the same arguments of Corollary 4.2 and Proposition 4.3, in order for $X$ to have singularities as mild as possible and, in any case, only $A_{1}$ singularities, it suffices to choose $D_{1}$ and $D_{2}$ smooth and meeting transversally. We see that such a choice is indeed possible. For a cover as in Theorem 6.1 (1), $D_{1} \sim(2 m-e+1) f$ and $D_{2} \sim 4 C_{0}+(2 e+2) f$. Then we choose $D_{1}$ as the union of $2 m-e+1$ different fibers. The divisor $4 C_{0}+(2 e+2) f$ is base-point-free if $e=0,1$ and if $e=2$ is $\left(3 C_{0}+6 f\right)+C_{0}$, with $3 C_{0}+6 f$ base-point-free and $\left(3 C_{0}+6 f\right) \cdot C_{0}=0$. Thus by Bertini $D_{1}$ and $D_{2}$ can be chosen smooth and intersecting transversally. Finally, in Theorem 6.2. $D_{1}$ and $D_{2}$ are the union of distinct smooth lines belonging to the two fibrations of the ruled surface $\mathbf{F}_{0}$. Note that, if $X$ is regular, one can construct $X$ with worse singularities allowing $D_{1}+D_{2}$ to have worse singularities.

\section{Non-EXISTENCE OF SIMPLE CYCLIC COVERS}

In Sections 4, 5 and 6, we have seen that quadruple cyclic canonical covers of smooth surfaces of minimal degree are never simple cyclic. This situation does not only hold for covers of degree 4, but is more general. For instance, if $X$ is regular and $W$ is a surface of minimal degree, whether smooth or singular, the authors proved in GP2 the non-existence of simple cyclic canonical covers of degree larger than 3.

In the next theorem we prove the non-existence of simple cyclic canonical covers of degree larger than or equal to 3 when $X$ is an arbitrary surface of general type and $W$ is a smooth rational normal scroll.

Theorem 7.1. Let $W$ be a smooth rational normal scroll, and let $X \stackrel{\varphi}{\longrightarrow} W$ be a canonical cover of degree $n$. If $X \stackrel{\varphi}{\longrightarrow} W$ is a Galois cover and $n \geq 3$, then $\varphi$ is not simple cyclic.

Proof. Let us assume $\varphi$ is simple cyclic. Then

$$
\varphi_{*} \mathcal{O}_{X}=\mathcal{O}_{W} \oplus L^{-1} \oplus \cdots \oplus L^{-n+1} .
$$

Recall that $W$ is isomorphic to $\mathbf{F}_{e}$. On the one hand

$$
\omega_{X}=\varphi^{*} \mathcal{O}_{W}(1)=\varphi^{*} \mathcal{O}_{W}\left(C_{0}+m f\right)
$$


with $m \geq e+1$. On the other hand,

$$
\omega_{X}=\varphi^{*}\left(\omega_{W} \otimes L^{-n+1}\right)=\varphi^{*}\left(\mathcal{O}_{W}\left(-2 C_{0}-(e+2) f\right) \otimes L^{-n+1}\right) .
$$

Thus $\varphi^{*} L^{-n+1}=\varphi^{*} \mathcal{O}_{W}\left(3 C_{0}+(m+e+2) f\right)$, so that $L^{-n+1}$ and $\mathcal{O}_{W}\left(3 C_{0}+(m+e+2) f\right)$ are numerically equivalent in $W$. Since $W$ is a Hirzebruch surface, $L^{-n+1}=\mathcal{O}_{W}\left(3 C_{0}+(m+e+2) f\right)$, and 3 and $m+e+2$ are both multiple of $n-1$. Since $n \geq 3$ by assumption, this makes $n=4$. Then, Theorems 6.1 and 6.2 tell that $\varphi$ is not simple cyclic. This can also be seen directly as follows: since $\varphi$ is induced by the complete series of $\mathcal{O}_{W}\left(C_{0}+m f\right)$, then $\mathcal{O}_{W}\left(\left(m-\frac{1}{3}(m+e+2)\right) f\right)$ should be non-effective; this together with $m \geq e+1$ implies $e<0$, a contradiction.

After Theorem 7.1 we now summarize the status of the existence of simple cyclic canonical covers in the following theorem. To see the scope of the result, we remark that Theorem 7.2 implies the non-existence of Galois canonical covers of prime degree $p$ of smooth scrolls, $\mathbf{P}^{2}$ or the Veronese surface, if $p \geq 5$. If in addition $X$ is regular, the next theorem assures that, if $p \geq 5$, then there are no Galois canonical covers of prime degree $p$ of any surface of minimal degree.

Theorem 7.2. Let $W$ be a surface of minimal degree, not necessarily smooth, and let $X \stackrel{\varphi}{\longrightarrow} W$ be a Galois canonical cover. If $X$ is regular or $W$ is smooth, and if $\varphi$ is simple cyclic, then deg $\varphi \leq 3$.

Proof. If $X$ is regular, the result follows from [GP2, Corollary 3.2]. So we will assume that $X$ is irregular and $W$ smooth. The surface $W$ cannot be isomorphic to $\mathbf{P}^{2}$, for if it were, since $\varphi$ is simple cyclic, $\varphi_{*} \mathcal{O}_{X}$ would split completely, and so $X$ would be regular. Thus $W$ is a smooth rational normal scroll. Then we conclude that $\operatorname{deg} \varphi \leq 3$ by applying Theorem 7.1 .

Corollary 7.3. Let $W$ be a surface of minimal degree, not necessarily smooth, and let $X \stackrel{\varphi}{\longrightarrow} W$ be a Galois canonical cover. If $X$ is regular or $W$ is smooth and $\varphi$ is a Galois canonical cover of prime degree, then $\operatorname{deg} \varphi \leq 3$.

Proof. If $\operatorname{deg} \varphi=p$ is prime, then $G$ is cyclic of order $p$, and the stabilizer of any $x \in X$ is either $\{i d\}$ or $G$, so $\varphi$ is simple cyclic.

These results hint towards a positive solution to the following very interesting question regarding Galois canonical covers of prime degree larger than 3:

Question 7.4. If $X \stackrel{\varphi}{\longrightarrow} W$ is a Galois canonical cover of prime degree, is deg $\varphi \leq 3 ?$

\section{ACKNOWLEDGEMENTS}

We thank N. Mohan Kumar for generously sharing his time for some useful discussions and comments. We also thank Dale Cutkosky for some useful discussions and Rita Pardini for helpful comments.

\section{References}

[Ba] L. Bădescu, Algebraic Surfaces Universitext. Springer-Verlag, New York, 2001. MR1805816 (2001k:14068)

[Be] A. Beauville, L'application canonique pour les surfaces de type general, Inventiones Math. 55 (1979), 121-140. MR553705 (81m:14025) 
[BS] M.C. Beltrametti and T. Szemberg, On higher order embeddings of Calabi-Yau threefolds, Arch. Math. (Basel) 74 (2000), 221-225. MR.1739501(2001d:14039)

[Ca] F. Catanese, On the moduli spaces of surfaces of general type. J. Differential Geom. 19 (1984), no. 2, 483-515. MR755236 (86h:14031)

[GP1] F.J. Gallego and B.P. Purnaprajna, Very ampleness and higher syzygies for Calabi-Yau threefolds, Math. Ann. 312 (1998), 133-149. MR.1645954 (99g:14048)

[GP2] F.J. Gallego and B.P. Purnaprajna, On the canonical ring of covers of surfaces of minimal degree, Trans. Amer. Math. Soc. 355 (2003), 2715-2732. MR.1975396 (2004b:14069)

[GP3] F.J. Gallego and B.P. Purnaprajna, Classification of quadruple canonical covers: Galois case, C. R. Math. Acad. Sci. Soc. R. Can. 26 (2004), 45-50. MR2055225 (2005a:14016)

[GP4] F.J. Gallego and B.P. Purnaprajna, Classification of quadruple Galois canonical covers, II. Journal of Algebra. 312 (2007), 798-828. MR2333185

[Gr] M.L. Green, The canonical ring of a variety of general type, Duke Math. J. 49 (1982), 1087-1113. MR683012 (84k:14006)

[HM] D. Hahn and R. Miranda, Quadruple covers of algebraic varieties, J. Algebraic Geom. 8 (1999), 1-30. MR:1658196 (99k:14028)

[Ho] E. Horikawa, Algebraic surfaces of general type with small $c_{1}^{2}$, I, Ann. of Math. (2) 104 (1976), 357-387. MR0424831 (54:12789)

[Ko] K. Konno, Algebraic surfaces of general type with $c_{1}^{2}=3 p_{g}-6$, Math. Ann. 290 (1991), 77-107. MR.1107664 (92i:14039)

[No] M. V. Nori, Zariski's conjecture and related problems, Ann. Sci. École Norm. Sup. (4) 16 (1983), 305-344. MR732347 (86d:14027)

[OP] K. Oguiso and T. Peternell, On polarized canonical Calabi-Yau threefolds, Math. Ann. 301 (1995), 237-248. MR1314586 (96b:14050)

[Pe] U. Persson, Double coverings and surfaces of general type, Algebraic Geometry, Lect. Notes Math. vol. 687 (1978), 168-195. MR527234 (80h:14017)

Departamento de Álgebra, Facultad de Matemáticas, Universidad Complutense de MADRID, 28040 MADRID, SPAIN

E-mail address: gallego@mat.ucm.es

Department of Mathematics, 405 Snow Hall, University of Kansas, Lawrence, Kansas $66045-2142$

E-mail address: purna@math.ku.edu 